\title{
"L'œuvre poétique en mouvement: une approche intertextuelle de la poésie de Raymond Queneau"
}

Amal Abdel-Sattar

La notion d'intertextualité, dès sa naissance, ne s'avère pas en tant qu'abordable; elle subit, dès lors, une jonchée de changements qui la rend, en passant le temps, plus docile à saisir, bien qu'elle reste parmi les questions littéraires les plus épineuses. Dans cette recherche nous essayons d'appliquer quelques éléments intertextuels au corpus afin de montrer que l'œuvre quenienne subit les mêmes changements que subit la littérature tout entière. L'ouvrage même de Tiphaine Samoyault était de grand profit pour nous: une certaine classification de la part de Samoyault nous mène nécessairement à découvrir dans un grand nombre de poèmes queniens des empreintes et des traces d'autres écrivains. Cette taxinomie bien détaillée met à nos yeux le projet même d'un Queneau dont l'intention poétique et esthétique l'a exempté de tomber dans le plagiat.

\section{Historique et définitions variables}

Officiellement, le terme d'intertextualité était composé et introduit par Julia Kristeva, dans deux articles parus dans la revue Tel Quel et repris ensuite dans son ouvrage publié en 1969, Sémiotikè, Recherches pour une sémanalyse.

Dans le premier article, paru en 1966 et intitulé "Le mot, le dialogue, le roman", la notion a connu sa naissance: "tout texte se construit comme une mosaïque de citations, tout texte est absorption et transformation d'un autre texte. A la place de la notion d'intersubjectivité s'installe celle d'intertextualité, et le langage poétique se lit, au moins comme double." 1

Le deuxième article, "Le texte clos", avait doté d'une nouvelle perspective la théorie $\mathrm{du}$ texte qui s'est défini, dès lors, comme "un appareil translinguistique qui redistribue l'ordre de la langue." ${ }^{2}$ Le terme d'intertextualité est devenu parallèlement lié au terme de productivité, ce qui veut dire que "dans l'espace d'un texte, plusieurs énoncés, pris à d'autres textes, se croisent et se neutralisent." ${ }^{3}$

A la suite de Kristeva, le terme a été repris par plusieurs théoriciens de Tel Quel dans l'ouvrage collectif intitulé Théorie d'ensemble. Philippe Sollers l'a formulé ainsi: "tout texte se situe à la jonction de plusieurs textes dont il est à la fois la relecture, l'accentuation, la condensation, le déplacement et la profondeur." ${ }^{4}$

Dans tout texte le mot introduit un dialogue avec d'autres textes: c'est bien l'idée que Julia Kristeva emprunte à Bakhtine et qui entraîne la naissance de la notion d'intertextualité. Le dialogisme de Mikhaïl Bakhtine s'avère en quelque sorte l'équivalent de la polyphonie où, dans un texte donné, toutes les voix résonnent d'une façon égale. ${ }^{5}$ Cela implique, de son tour, l'idée de l'altérité, où le texte est toujours en mouvement, où on entend parler dans le texte des mots autres, des mots des autres.

Roland Barthes confirme que tout texte est un tissu nouveau de "citations inconscientes ou automatiques, données sans guillemets. ${ }^{66}$ La notion d'intertexte 
n'est pas, donc, une autorité, c'est simplement, selon Barthes, un souvenir circulaire, et la dimension du mot inter-texte signifierait plutôt "l'impossibilité de vivre hors du texte fini." ${ }^{7}$ La tâche de la réception littéraire, toute différente de la critique traditionnelle, portera sur un double aspect d'étude du texte: une intertextualité de surface, étudiant le fait de l'écriture (étude typologique et formelle des gestes de reprise), et une intertextualité en profondeur, étudiant les relations nées des contacts des textes entre eux.

Le texte comme un effet de lecture, accorde l'intertextualité au concept de la réception. L'intertexte représente un phénomène qui oriente le lecteur du texte, et qui est "le contraire de la lecture linéaire." ${ }^{8}$ Le lecteur est le maître, c'est lui qui dirige la lecture et sur sa propre mémoire s'appuie la perception, dans un texte, d'analogies avec d'autres textes.

Pour Gérard Genette, l'intertextualité se définit comme la présence effective d'un texte dans un autre texte. Il la distingue de l'hypertextualité, par laquelle un texte peut dériver d'un texte antérieur sous la forme notamment de la parodie et du pastiche. Selon lui, la transtextualité est l'objet de la poétique dont relève chaque texte et dont bifurquent cinq types:

- l'intertextualité qui montre une relation de coprésence entre deux ou plusieurs textes (A est présent avec B dans le texte B), c'est bien évident dans les pratiques de la citation, du plagiat et de l'allusion;

- la paratextualité qui est la relation qu'entretient le texte avec son paratexte, c'est-à-dire le sous-titre, la préface, etc.;

- la métatextualité qui décrit la relation de commentaire unissant un texte au texte dont il parle;

- l'hypertextualité qui relève de la dérivation d'un texte (B dérive A mais A n'est pas effectivement présent dans B);

- l'architextualité qui détermine le statut générique du texte. Genette a choisi comme titre pour son ouvrage le mot Palimpsestes, qui désigne ces affinités cachées ou apparentes liant un texte à un ou plusieurs autres textes. Ce titre renvoie au "manuscrit effacé et récrit qui laisse paraître, en filigrane, des traces variables du texte antérieur."9

Antoine Compagnon s'intéresse à la pratique intertextuelle dominante, c'est-à-dire la citation. Il voit que toute écriture est collage et glose, citation et commentaire. ${ }^{\mathbf{1 0}}$ Dans ce sens, le travail de l'auteur ne dépasse pas la réinsertion et le recyclage. En dépit de cela, l'absorption dans un texte d'un texte antérieur n'est pas totale et laisse voir les jointures: le point de départ d'un auteur était le point d'arrivée d'un autre. On admet, donc, que tout texte est second, il ne rompt pas avec le texte qui le précède et ne rompra jamais avec le texte qui le suit.

Aux années soixante-dix et quatre-vingts, la notion a subi un assouplissement qui la rend beaucoup plus flexible. Laurent Jenny voit que le texte parle une langue dont le vocabulaire est la somme des textes existants. L'examen de remodelage que subit un texte implique un travail visant à préciser les modalités des transformations dans 
l'intertexte. La "greffe"11 intertextuelle, pourrait être sondée d'après la paronomase, par exemple, qui consiste à reprendre un texte selon les sonorités; l'amplification, qui consiste à transformer le texte originel par développement de ses possibilités sémantiques; l'hyperbole qui transforme un texte par l'expansion formelle; le changement de niveau de sens;... etc.

Donc, l'intertextualité apparaît comme détournement culturel, comme réactivation du sens ou comme mémoire des sujets. ${ }^{12}$ Michel Schneider a utilisé la psychanalyse pour déceler les rapports du moi et de l'autre dans l'activité de lecture-écriture. Il emploie des expressions qui prennent la relève du terme d'intertextualité: "un texte pour l'autre" désigne le plagiat, "un texte sous l'autre" le palimpseste et "un texte comme l'autre" le pastiche. Un texte, selon lui, n'existe pas tout seul, il est chargé de mots et d'idées plus ou moins consciemment volés et il semble toujours d'y déceler un sous-texte. ${ }^{13}$

Le rôle d'un "lecteur-moderne" fait jour. L'opération intertextuelle exige la présence de deux pôles: le pôle artistique, représenté par l'œuvre littéraire, et le pôle esthétique, qui se produit de la consommation variable de la part du lecteur. ${ }^{14}$ Il est question, ici, d'un lecteur implicite, c'est grâce à lui que l'œuvre ne sera pas censée finie. C'est un lecteur-interprète qui est exprimé par plusieurs formules chez les grands écrivains: c'est un super-lecteur, chez Riffaterre ${ }^{15}$, qui le voit celui qui arrive toujours à un point compliqué dans le texte littéraire, ce point appelé la vérité stylistique est où l'on peut découvrir le sens chargé de codes au moyen de ce lecteur.

Stanley Fish le nomme le lecteur informé, c'est lui qui absorbe le texte littéraire grâce à sa connaissance sémantique très intense, il relie toujours son expérience cognitive au déchiffrement du texte. Erwin Wolf ${ }^{16}$ l'appelle le lecteur intentionné, celui qui préexiste dans la pensée de l'auteur. Il incarne toujours les conceptions et les coutumes du public contemporain, c'est au moyen de celui-là que l'auteur s'adhère au public. ${ }^{17}$

]]En un mot, cet arrière-plan nous est indispensable pour détecter le plus possible le mystère qui a couvert la notion d'intertextualité et qui semble faire le lecteur perdu dans ses coulisses. Au lieu de se sentir pris au piège de ses mystères, il prend part à la productivité du texte: le lecteur existe pour engendrer de nouvelles interprétations, il est complice dans la potentialité de l'œuvre littéraire. Dans les pages suivantes, nous allons faire une approche de ce que pourrait être un champ d'application du fait intertextuel, et comment exercer, dans la peau du lecteur, les pratiques intertextuelles ayant pour cible l'œuvre poétique de Queneau en tant que modèle de potentialité littéraire.

\section{A- Intertextualité et fusion des genres}

"Plus ou moins tous les livres contiennent la fusion de quelques redite comptée"18, par cette parole de Mallarmé nous introduisons cette partie dans la mesure où s'entremêlent plusieurs genres littéraires qui co-existent ensemble dans notre corpus. 
Palimpsestes de Gérard Genette a donné libre main aux critiques contemporaines de distinguer, dans toute œuvre, entre deux types de pratiques intertextuelles. Les premières inscrivent une relation de coprésence (le texte A est présent dans le texte $\mathrm{B}$ ) et les secondes une relation de dérivation (A est repris et transformé dans B: dans ce cas, il s'agit de pratique hypertextuelle).

De plus, il nous semble parler des opérations que subit le texte de départ dans son itinéraire jusqu'au texte d'accueil. Ces opérations, qui ne dépassent pas les sens déjà indiqués de l'intertexte et de l'hypertexte, se limitent à deux actions essentielles: l'intégration et le collage. Nous nous inspirons la division suivante des opérations intertextuelles du schéma qu'a suivi Tiphaine Samoyault dans le chapitre deuxième de son ouvrage estimable L'intertextualité, Mémoire de la littérature.

\section{a. l'intégration}

Cette opération consiste, pour les textes qui absorbent, à installer un ou plusieurs textes antérieurs dans le texte actuel. Cela prend plusieurs figures dont:

\section{L'intégration-installation}

Comme la citation marquée par deux guillemets ou bien par l'italique. Ces marques permettent de localiser l'intertexte et suppriment la mise à distance. Mais, le lecteur qui ne connaît pas la source de cette citation se trouve placé en situation d'ignorance et d'infériorité face au destinateur.

La référence précise révèle aussi l'intégration-installation. Il peut s'agir d'un titre (en italique) et d'un nom d'auteur, d'un nom de personnage et d'un nom d'auteur,... etc.

\section{L'intégration-suggestion}

Elle suppose la présence de l'intertexte sans la développer. Cela exige une étendue du savoir du lecteur et de son imagination des rapprochements. Nous pouvons en trouver des exemples dans la référence simple dont la mention d'un nom (d'auteur, de mythe, de personnage) ou d'un titre peut renvoyer à de multiples textes. Dans ce cas, l'intertexte se dilue, il devient presque interminable. Pour l'allusion, elle peut être confondue avec un certain nombre d'indices textuels vagues avec la référence simple, sans assignation particulière.

\section{L'intégration-absorption}

Dans cette opération, le texte absorbe l'intertexte sans même le suggérer au lecteur. Aucune marque distinctive ne permet de l'identifier avec évidence. Citons sous cette rubrique l'impli-citation ${ }^{19}$, un terme forgé par des critiques de l'œuvre de Georges Perec désignant la citation implicite, entièrement fondue dans le texte d'accueil. En ce cas, l'auteur n'indique jamais, par un signe quelconque, de quels passages il s'agit, ni d'où ils proviennent. Bien que la citation non marquée, elle se distingue du plagiat par les possibilités narratives (autobiographiques ou fictionnelles) qu'elle permet 
dans le texte d'accueil et dont la lecture ouverte à côté du savoir et de l'enquête peuvent effacer l'intertexte.

Bernard Magné distingue deux types d'impli-citation: la simple, qui se contente de supprimer les signes de changement d'énonciation (guillemets, alinéa, notes) permettant à un énonciateur de s'attribuer le discours d'un autre; et la complexe, qui signale un changement d'énonciation; mais "le locuteur rapporté explicite n'est pas l'auteur réellement cité"20: l'acte de citer n'est pas dissimulé mais un énonciateur fictif s'est approprié le discours du véritable énonciateur qui n'est pas mentionné. Pour ne pas se prendre en tant que plagiat, l'auteur, en hors-texte, à la fin, dans le post-scriptum signale la préexistence de telle ou telle citation chez un véritable auteur auquel il emprunte la sienne. Les termes de vol et de fraude attribués au plagiat déplacent la question du littéraire vers le juridique.

\section{b. Le collage}

Dans ces opérations, le texte principal n'intègre plus l'intertexte mais il le pose à côté de lui. Le lien qu'entretient le matériau collé avec le reste du texte ne dépasse pas l'une des formes suivantes:

\section{Au dessus du texte}

L'épigraphe, détachée du texte qu'elle surplombe et introduit, est généralement constituée d'une citation suivie de la référence à son auteur et / ou au texte dont elle est issue. ${ }^{21}$ Le texte rompt ensuite avec

montré cet aspect de collage quand nous citons l'épigraphe de Boileau dans Chêne et Chien I:

"...Quand je fais des vers, je songe toujours à dire ce qui ne s'est point encore dit en notre langue $(. .)$.$" .$

Si l'épigraphe est détachée, dans la forme, du texte où il est intégré, elle lui est liée par le sens. Un texte peut donc exister dans un autre texte sans lui être intrus. Le sens garantit au texte intégré la coexistence avec le texte accueillant.

\section{Au milieu du texte: intégration de documents}

Il arrive que les textes littéraires installent en leur centre un matériau intertextuel sans l'absorber. Les citations installées ne sont pas fondues dans le texte mais isolées en paragraphes distincts, séparées par des blancs. Leur sens ne se saisit donc pas en continuité et en uniformité avec le reste du texte mais se crée aussi, comme avec l'épigraphe, dans la dispersion. ${ }^{22}$

De tout ce qui précède et comme corollaire de ces opérations d'emprunts il nous faut mettre l'accent sur deux marques caractérisant fortement l'intertexte: la discontinuité; même avec l'absorption par le texte d'accueil, la citation ouvre le texte 
sur une extériorité et une altérité qui perturbe son unité et le place du côté de la dispersion. La deuxième marque est la collectivité des œuvres qui ouvrent le texte sur un examen de jeux de la mémoire littéraire.

En cela, les idées n'appartiennent à personne, "elles circulent, volent, se dispersent et se posent, au gré d'un vent dont il faut mesurer l'orientation." ${ }^{23}$ Bien que tout soit dit, le travail de l'écrivain tâche de débiter ce qu'il veut à travers une conception purement intertextuelle qui nécessite un petit aperçu sur cette théorie récemment construite. Dans les quelques pages suivantes nous présenterons des modèles de l'expérience quenienne concernant ce sujet.

\section{La citation}

Elle pourrait être définie comme une "répétition d'une unité de discours dans un autre discours", ou "un énoncé répété et une énonciation répétante." ${ }^{24}$ C'est aussi, selon Tiphaine Samoyault, "la production d'un énoncé (le texte cité), qui se trouve arraché d'un texte d'origine (texte 1) pour être introduit dans un texte d'accueil (texte 2)." 25

Selon Antoine Compagnon, "toute écriture est collage et glose, citation et commentaire" $^{\prime 2}$. La citation s'inscrit en tant qu'emprunt littéral explicite, c'est-à-dire qu'elle se distingue par l'usage de marques typographiques spécifiques. Les guillemets, les italiques, l'éventuel décrochement distinguent le fragment emprunté, et leur absence transforme la citation en plagiat.

De cet aspect de réinsertion nous trouvons que l'œuvre poétique de Raymond Queneau en est pleinement animée:

"mon amour est mon paradis

le vert paradis des amours." (Chêne et chien, II, p.22)

Le vers est mis en valeur par l'italique, un petit effort de la mémoire peut mettre en évidence un autre vers de Baudelaire:

"-Mais le vert paradis des amours enfantines."27

Il ne s'agit pas du vers entier qu'on insère, mais l'emprunt est clair, l'italique fait échapper l'auteur à tel ou tel plagiat. Dans le même poème nous rencontrons une autre citation:

\section{"Je t'apporte l'enfant d'une nuit bitumée." (p.23)}

Cette fois le vers renvoie à un vers de Mallarmé dans un poème intitulé Don $d u$ poème:

"Je t'apporte l'enfant d'une nuit Idumée (...)."28

L'emprunt est fidèle, Queneau remplace le mot "Idumée" par le mot "bitumée", seulement les mots empruntés sont mis en italique. 
Dans les vers qui suivent le poète utilise l'emprunt d'une autre manière:

"je dévale la pente

dies irae dies illa

sic ibo ad astra." (Si tu t'imagines, IV, Vieillir, p.116)

Le latinisme ici distingue les mots empruntés des mots propres à l'écrivain. Cela s'avère comme une marque typographique essentielle caractérisant les mots latins. Le premier vers est une des célèbres proses du missel romain ("Jour de colère ce jour là"), chantée à l'office des morts. Le deuxième ("Ainsi je monterai jusqu'aux astres") renvoie au vers de Virgile dans son Énéide, IX ("Macte nova virtute, puer; sic itur ad astra"). ${ }^{29}$

Pour conclure, nous nous interrogeons sur ce que peut signifier un tel recours à la citation. Jean Starobinski voit à ce propos que la citation se montre comme une "conséquence de l'autodépréciation mélancolique." ${ }^{30}$ Nous opposons à cette vision une tendance d'appréciation de l'autre, c'est-à-dire que l'auteur dans sa recherche de citation, y inscrit en même temps une quête permanente d'un véritable modèle à suivre; il apprécie l'écrivain qu'il cite et auquel il emprunte les mots.

\section{La référence}

C'est une des opérations d'intégration-installation qui peut se définir comme un "emprunt non littéral explicite." ${ }^{31}$ Elle n'expose pas le texte cité, mais y renvoie par un titre, un nom d'auteur, de personnage,... Il nous semble nécessaire de noter qu'on peut avoir une certaine ambiguïté à classer la référence parmi les formes de l'intertextualité. Ce n'est pas le cas quand il s'agit de citation, car la relation de coprésence y est évidente; mais pour la référence la relation de coprésence peut être dans certains cas minimale.

La référence tend parfois à s'avérer au moyen d'un titre (en italique) et d'un nom d'auteur, d'un nom de personnage et d'un nom d'auteur, etc. Dans ce cas on l'appelle une référence précise.

Nous citons cet exemple extrait de Si tu t'imagines, dans lequel nous découvrons le titre d'un ouvrage inconnu, que le poète a mis en italique:

"La science de Dieu: le soleil c'est le diable comment expliquer une telle inversion?" (Chêne et chien, II, p.24)

Claude Debon nous donne une explication satisfaisante à ce propos quand elle renvoie ce titre à un ouvrage de Pierre Roux, l'un des "fous littéraires" auquel Queneau avait fait une large place dans son Encyclopédie des sciences inexactes. ${ }^{32}$ Dans le même poème le poète se donne la licence d'emprunter de l'ouvrage de Roux un passage entier, et pour éviter toute possibilité de se connaître en tant que plagiaire le poète met le passage emprunté entre deux guillemets:

\footnotetext{
"“ Son noyau est excrémentiel.
} 


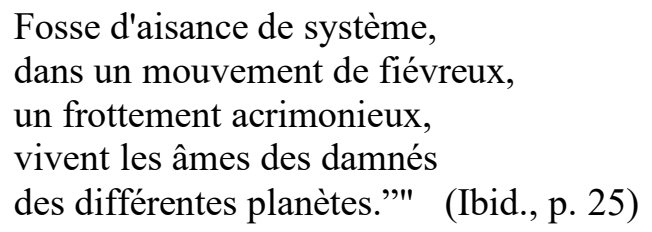

Un autre exemple est flagrant à ce propos; Queneau dote un de ses poèmes de ce titre significatif "To be or not to be". Il nous devient très évident que le poète fait référence au célèbre "Hamlet" de Shakespeare. Il se contente aussi de clore le poème d'une autre référence d' Hamlet $^{33}$ en faisant allusion à Shakespeare lui-même:

"jusqu'à ce qu'un poète anglais

cessant de le rimer champêtre

l'accord avec le néant."

(Battre la campagne, To be or not to be, p.464).

Le poète s'ingénie à insérer le titre d'un tel ou tel ouvrage dans ses poèmes, mais parfois cette insertion ne se distingue pas du reste des vers, c'est-à-dire que le poète ne la distingue pas d'une marque tels les italiques, par exemple, comme nous le voyons dans l'exemple suivant:

"amère amère victoire

le roseau qui n'a pas bougé."

(Battre la campagne, Le peuplier et le roseau, p.469).

"Amère victoire" c'est le titre d'un roman de René Hardy (1927). ${ }^{34}$ Nous trouvons que cet ouvrage figure dans la liste des lectures de R. Queneau. ${ }^{35}$ On ne sait pas dans le poème qu'il s'agit d'un titre de roman; mais la répétition de l'adjectif "amère" et la résonance que fait cette répétition mettent l'accent sur l'importance de cette structure. Autrement dit, la répétition de l'épithète "amère" remplace les italiques dans la précision de cette référence.

\section{L'allusion}

C'est une opération d'intégration-suggestion; elle renvoie à un texte antérieur sans marquer l'hétérogénéité autant que la citation. Elle dépend de l'effet de la lecture. ${ }^{36}$ Définie en tant qu"'emprunt non littéral non explicite" ${ }^{37}$, on ne saurait pas très exactement la différencier de la référence simple; mais l'allusion, pas pleinement visible, permettrait une connivence entre l'auteur et le lecteur qui parvient à l'identifier, voire à la suggérer.

Prenons ces vers extraits de Si tu t'imagines pour voir dans quelle mesure l'allusion était utilisée dans l'œuvre quenienne:

"Suivant un cours certain à travers les étoiles

jaillit le lait béni qui fonda la blancheur

trouée ainsi la nuit laisse couler sans voiles 
le galion incertain de l'angoisse et des pleurs."

(Si tu t'magines, Les Ziaux., I, Iris, p.44).

Claude Debon suggère certaines affinités liant cet extrait à "La chanson du mal aimé" d'Apollinaire. ${ }^{38}$ Voici l'extrait modèle d'Apollinaire:

"Voie lactée ô sœur lumineuse
Des blancs ruisseaux de Chanaan
Et des cœurs blancs des amoureuses
Nageurs morts suivrons-nous d'ahan
Ton cours vers d'autres nébuleuses."

Les vers précédents, de Queneau et d'Apollinaire, font allusion au mythe de la voie lactée qui a été formée, selon la mythologie, par des gouttes de lait de la déesse Junon $^{40}$, déesse latine du mariage, nourrissant Hercule enfant. Elle sera identifiée ensuite avec l'Héra grecque. ${ }^{41}$ Apollinaire prend la part du lion dans la liste des influences exercées sur Queneau. Ce n'est pas uniquement l'œuvre poétique d'Apollinaire dont on trouve les traces chez un Queneau, mais il s'agit cette fois de ses œuvres en prose: dans l'extrait suivant de Si tu t'imagines nous trouvons l'exemple dont un jeu de calembour:

"Nous lézards aimons les Muses

Elles Muses aiment les Arts

Avec les Arts on s'amuse

On muse avec les lézards."

(Si tu t'imagines, Les Ziaux., II, Muses et lézards, p. 52).

Dans La suite de Cendrillon ou le Rat et les six lézards, Apollinaire "bâtit également son récit sur le calembour lézards, les arts"..$^{42}$ Donc, nous trouvons dans l'ouvrage d'Apollinaire: "Et on peut admirer ici combien les surnoms populaires sont sensés puisque 'les Arts' étaient bien nommés, ayant été des lézards". ${ }^{43}$ Queneau emprunte à Apollinaire l'usage humoristique de ces deux mots. Dans La statue de plâtre, nous entendons un écho qui pourrait évoquer celui de Crépuscule d'Apollinaire dans Alcools $^{44}$, prenons d'abord le poème de Queneau:

"(...) D'un rêve né du crépuscule

Rêve de morts qu'on émascule (...)

(...) pour récompenser l'arlequin

De fleurs noires les étrangères (...)"

(Si tu t'imagines., L'instant fatal., I, La statue de plâtre, p. 78).

Observons le poème d'Apollinaire:

"Un charlatan crépusculaire

Vante les tours que l'on va faire (...)

(...) sur les tréteaux l'arlequin blême 
Salue d'abord les spectateurs $(\ldots)^{\prime 45}$

Selon Claude Debon, les deux poèmes ont la même atmosphère onirique et funèbre, les mots "crépuscule, crépusculaire, arlequin", les deux poèmes ont aussi des quatrains octosyllabiques. $^{46}$

L'œuvre d'Apollinaire représente la part considérable des influences sur Queneau, l'idée même de "Cimetière oublié" de Queneau se base sur un poème d'Apollinaire dans Alcools, intitulé "La maison des morts". ${ }^{47}$ Chez celui-ci, les morts se raniment, ils marchent en petite troupe avec les vivants pour se promener, il y avait parmi les morts et les vivants ceux et celles qui s'aimaient:

"Les morts avaient choisi les vivantes

Et les vivants

Les mortes." 48

Il y avait aussi des conversations, de la danse et du bonheur parmi eux. Au soir, il semble que la "troupe diminua[it] peu à peu / [et] [o]n se disait / [a]u revoir / [à] demain / [à] bientôt." ${ }^{49}$ Le poète restait seul avec les morts qui "s'en allaient tout droit / [a]u cimetière." ${ }^{50}$ Mais pour les vivants, ils "en gardaient le souvenir" ${ }^{51}$; car ils aimaient les morts, ils "[a]dmiraient maintenant / [1] eur puissance leur richesse et leur génie" ${ }^{52}$, il leur vaut mieux "d'avoir aimé un mort ou une morte / On devient si pur qu'on en arrive." ${ }^{53}$ Pour Queneau, dans son Cimetière oublié, les morts se révoltent; cette fois, ils ne sortent pas en troupe, mais ils sont syndiqués:

\section{"Les morts révoltés \\ les morts syndiqués \\ sortent du cimetière avec leurs crânes délavés."}

(Battre la campagne., Cimetière oublié, p. 473).

Cette fois, ils parlent et ont de longues conversations, mais c'était avec un seul vivant, le maire:

"ils s'étaient syndiqués et le maire leur a parlé

colloque discours et rencontre au sommet." (Loc.cit.)

Les morts d'Apollinaire "s'en allaient tout droit au cimetière", mais ceux de Queneau étaient conduits par un garde champêtre:

"le soir le garde champêtre les a conduits au cimetière

et puis il a fermé la porte

d'un quadruple tour d'une lourde clé." (Loc.cit.)

Cela diffère un peu quand on parle du cimetière d'Apollinaire qui était dès le début de la scène désert:

"J'étais entré pour la première fois et par hasard 
Dans ce cimetière presque désert." 54

Pour celui de Queneau, il s'agit d'un cimetière oublié et abandonné, mais c'était après la révolte du royaume des morts et après que le garde champêtre avait fermé la porte du cimetière à jamais:

"depuis ce jour-là le cimetière est abandonné on ne visite plus le cimetière." (Loc.cit.)

La liste des influences sur Queneau est animée de plus d'une voix, comme celle d'Alfred de Vigny:

"c'est pourquoi l'on entend dans les forêts voisines parfois au fond des bois un petit air de cor."

(Battre la campagne, Le chant des bois, p.447)

On entend l'écho de ces vers dans Poèmes antiques et modernes, un recueil de poèmes d'Alfred de Vigny, dans un poème qui prend le titre "Le cor" ${ }^{55}$ :

"J'aime le son du Cor, le soir, au fond des bois." ${ }^{56}$

Dans d'autres vers Queneau cite Baudelaire ${ }^{57}$, il y fait allusion:

"Reprends donc ta sarclette, ô mort vieux capitaine fais ton petit métier."

(Ibid., Un moment de repos, p. 490)

Le vers baudelairien est le suivant:

"O Mort, vieux capitaine, il est temps! Levons l'ancre." 58

Dans les deux vers précédents, la mort s'avère en tant que "vieux capitaine", Baudelaire lui demande de lever l'ancre, Queneau lui demande de faire son métier, deux métaphores qui indiquent la rigueur et la fatalité de l'action.

L'allusion semblerait claire quand il s'agit d'un titre, c'est le cas ici de Shakespeare auquel le poète emprunte le titre d'un ouvrage très célèbre, c'est "Songe d'une nuit d'été" qui devient en passant à Queneau "Songe d'une nuit d'hiver"! (B.L.C., p.445); le remplacement, ici, d'un mot n'empêche pas le lecteur de suggérer et de renvoyer le titre inventé au titre originel.

\section{L'impli-citation}

Cela se pose sous la rubrique des opérations d'intégration-absorption que nous avons déjà signalé dans les pages précédentes. Le terme, lui-même, d'impli-citation est un terme forgé par des critiques de l'œuvre de Georges Perec. Il désigne, ce terme là, la citation implicite, entièrement fondue dans le texte d'accueil, mais sans 
aucune indication de l'auteur, par un signe quelconque, de quel passage il s'agit, ni d'où ces extraits proviennent. ${ }^{59}$ Il y'en a deux types: la simple et la complexe. La première se contente de supprimer les signes de changement d'énonciation (guillemets, alinéa, notes) permettant à un énonciateur de s'attribuer le discours d'un autre au "nez et à la barbe" ${ }^{60}$ du lecteur. La deuxième signale un changement d'énonciation, mais le locuteur rapporté explicite n'est pas l'auteur réellement cité; dans ce cas, l'acte de citer n'est pas dissimulé, mais un énonciateur fictif s'est approprié le discours du véritable énonciateur qui n'est pas mentionné. ${ }^{61}$ Dans les deux types, ce qui reste caché est toujours le nom de l'énonciateur effectif qu'on pourrait dévoiler au moyen du hors-texte: dans le post-scriptum, par exemple.

Dans le premier type de l'impli-citation, Queneau nous donne l'exemple suivant: "ô promeneur urbain arrête un peu le bras."

(Battre la campagne, Les ares verts, p. 443)

Claude Debon signale, à ce propos, qu'il s'agit d'une "reprise partielle du premier vers de l'élégie de Ronsard, 'contre les bûcherons de la forêt de Gastine': 'Ecoute, bûcheron, arrête un peu le bras!"'62

Ici, le poète mêle les paroles de Ronsard aux siennes, il s'approprie celles-là; donc, il s'avérerait très difficile de les séparer les unes des autres et de distinguer le véritable énonciateur implicite. Le changement d'énonciation dans certains cas, chez Queneau, semblerait très clair. Le poète n'utilise pas les guillemets pour marquer le changement de l'énonciation, l'italique est parfois utilisé pour augmenter l'ambiguïté de la citation. Il est évident que les mots insérés dans le passage ne sont pas les mots propres du poète, leur énonciateur effectif est, lui-même aussi, inconnu; le horstexte, également, ne donne aucune indice sur le passage ou l'auteur desquels ces mots sont empruntés:

\section{"Insulté par ces cris sauvages,}

l'astre brillant de l'univers (...)"

(Si tu t'imagines, Chêne et chien, II, p. 25)

L'emploi de l'italique dans les vers précédents n'est pas justifié de la part du poète, cela montre que ces mots peuvent être lancés de la bouche d'un autre dont l'identité reste cachée.

Dans un autre exemple, le changement de l'énonciation devient plus clair:

"les vers vides c'est pour les chiens

les verres pleins c'est pour les chrétiens."

(Si tu t'imagines, Chêne et chien, III, p.34)

C'est à l'ambiguïté de l'emploi de l'italique que s'ajoute celle du rythme et de la musique de ces vers, qui donnent l'apparence d'un dicton! ${ }^{63}$ Il nous semble parfois que le poète avait inventé ces mots délibérément et qu'il avait l'intention de prendre son lecteur au piège. Il voudrait nous montrer l'une de ses possibilités intarissables d'inventer et de créer les mots, même s'ils paraissent ceux d'un autre auteur! 
"la fesse tremble comme le lait caillé dans le bol d'un Bédouin."

(Ibid., p. 36)

Dans cet exemple, l'italique ne couvre pas le vers entier. Nous, lecteurs, sommes tout à fait perturbés devant ce changement typographique qui entraîne un changement d'énonciation, le véritable énonciateur, qu'il soit Queneau ou non, reste anonyme.

\section{L'épigraphe}

C'est une opération de collage ${ }^{64}$, elle se montre détachée du texte qu'elle surplombe, et par une sorte de recyclage elle figure dans un autre texte dans lequel l'épigraphe se pose au-dessus. L'épigraphe suggère une séparation, grâce au blanc qui dissocie l'intertexte et le texte, et une réunion, puisque le texte s'approprie les qualités et le renom d'un auteur ou d'un texte précédent. Par conséquent, la place de l'épigraphe, en exergue au-dessus du texte, suggère la figure généalogique. ${ }^{65}$

Le mot "exergue" attribué à "épigraphe", c'est-à-dire la mise en exergue, ne passe pas sans paraitre aux yeux du lecteur en tant que perplexe. C'est à cause de sa signification littéralement faite, hors d'œuvre, qu'on pourrait tomber dans l'embarras. C'était exactement ce qui s'est imposé à Gérard Genette qui s'est contenté de signaler que "l'exergue est ici plutôt en bord d'œuvre, généralement au plus près du texte $[\ldots] .{ }^{166}$

Nous avons dans notre corpus deux épigraphes considérables: la première, le poète l'utilise pour introduire Chêne et chien; il y a mis son projet d'écriture:

"...Quand je fais des vers, je songe toujours à dire ce qui ne s'est point encore dit en notre langue. C'est ce que j'ai principalement affecté dans une nouvelle épître... J'y conte tout ce que j'ai fait depuis que je suis au monde. J'y rapporte mes défauts, mon âge, mes inclinations, mes mœurs. J'y dis de quel père et de quelle mère je suis né...

\section{Boileau". (Chêne et chien, I, p. 5)}

Queneau admire Boileau, il le qualifie de "grand poète"67, pour cette citation, il était question de l'épître $X$ de Boileau ${ }^{68}$. Selon Michèle Hanoosh, "(...) la signification particulière de la citation en épigraphe dépend de la relation avec son nouveau contexte." ${ }^{69}$ Et comme nous l'avons vu, il n'y a aucun désaccord entre le plan de s'épancher de Boileau et celui de Queneau, les deux visent à se dire, à tout dire.

Dans une autre épigraphe, de Chêne et chien II, le plan de tout dire qui a précédé s'avère beaucoup plus fructueux:

"To Infancy, o Lord, again I come

That I my Manhood may improve.

TRAHERNE." 70 
"A l'enfance, ô Seigneur, je reviens afin d'améliorer mon âge viril." Donc, il est question maintenant d'une quête purgative. C'est-à-dire que l'épigraphe qui a introduit la deuxième partie de Chêne et chien, commence par ces vers:

"Je me couchai sur un divan

et me mis à raconter ma vie." (loc.cit.).

Celle-ci nous mène à concevoir le plan cathartique que le poète a entamé. La citation empruntée à Traherne et réinsérée dans un autre contexte ne rompt pas avec la linéarité du texte d'accueil; l'intertexte y est homogène dans son ensemble.

\section{Parodie et pastiche}

C'est en parlant des œuvres qui peuvent s'inscrire dans une sorte de co-présence, Genette a souligné une autre forme de la co-existence des ouvrages multiples, celle de la dérivation. Ce qu'on peut plus nommer hypertextualité qu'intertextualité.

\section{A (hypotexte) $\longrightarrow$ B (hypertexte)}

Cette opération implique une transformation (parodie) ou une imitation (pastiche) du texte antérieur ou l'hypotexte (texte de départ), que l'hypertexte (texte d'accueil) évoque d'une manière ou d'une autre sans le citer directement.

Le mot parodie formé de ôdè, c'est le chant; para: "le long de", "à côté". ${ }^{71}$ C'est donc le fait de chanter à côté, de chanter faux, dans une autre voix ou sur un autre ton; c'est aussi déformer ou transposer une mélodie. ${ }^{72}$

En ce sens, l'hypotexte sera détourné pour le moquer. Dans le petit Robert c'est "une imitation burlesque d'une œuvre sérieuse". La valeur de la parodie pourrait être d'une visée ludique et subversive quand il s'agit de détourner l'hypotexte pour le moquer, cela pourrait être aussi pour l'admirer.

Prenons Les fables de La Fontaine comme modèle; la fable est un "récit qui, en général, raconte une anecdote à la troisième personne (...) (Ce récit est) bâti sur un système fondamentalement allégorique. ${ }^{173}$

Dans Le loup et l'agneau de La Fontaine "la raison du plus fort est toujours la meilleure" ${ }^{74}$ De ce principe, l'histoire commence par une scène très sereine et pacifique d'un agneau qui "se désaltérait / Dans le courant d'une onde pure". ${ }^{75}$

A ce moment là, survient un loup à jeun qui cherchait à manger. Il accusait le pauvre agneau d'avoir troublé son breuvage et que celui-ci serait châtié de sa témérité. Le loup emporte l'agneau et puis le mange au fond des forêts. Le plus fort présente toujours des prétextes pour donner légalité à ses actes abusifs.

L'image sera renversée chez Queneau, cette fois le plus fort est l'agneau: le titre même du poème est renversé, il s'agit de L'agnaeu et le loup. La scène commence, 
avec la même sérénité, par le loup qui broutait dans le buisson, c'est "un loup de la belle espèce / il boit aussi l'eau claire / du ru pur." (Battre la campagne, p. 438). La scène paisible sera interrompue par une bête sauvage, "un agneau de la même espèce" aussi, qui pose la question suivante au pauvre loup:

"pourquoi, dit-il, troubler

mon ru pur?" (Battre la campagne, L'agneau et le loup, p. 439)

Le loup voudrait s'enfuir devant la rage de l'agneau, mais celui-ci se met à cogner le loup, un peu de sang coule sur l'herbe, le loup s'enfuit et l'agneau triompha. La scène est moins sanglante que celle de La Fontaine; Queneau ajoute de l'humour à l'histoire dans quelques reprises:

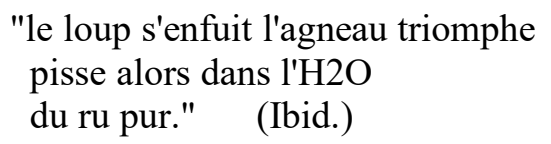

Le poète remplace le mot "eau" par son équivalent scientifique, l'H2O. La cocasserie du poème ne se limite pas à cette utilisation, mais aussi tout le poème ne respecte ni la rime ni le mètre comme celui de La Fontaine. Il serait mieux de conclure par cette conséquence qui voit que "le meilleur parodiste est toujours audessous de son modèle." ${ }^{176}$

Les deux gymnospermes c'est le titre d'un poème qui raconte l'histoire de deux plantes, le lichen et la fougère, qui s'aimaient très fort et vivaient heureusement jusqu'au au moment où la fougère décide de s'en aller, de partir en voyage:

\author{
"mais l'un d'eux s'ennuyait \\ s'ennuyait âprement (...) \\ La fougère un beau soir \\ prit son automobile \\ pour (...) s'en aller à la ville".
}

(Battre la campagne, Les deux gymnospermes, p. 472)

Après avoir découvert le cosmos, la fougère est revenue, mais elle trouvait que le lichen est mort "de douleur et d'amour" (ibid.). La fougère en pleurant bien fort ressuscitait le corps mort du lichen qui aimait de nouveau la belle fougère. Ils avaient des enfants, des petites gymnospermes et vivaient dans le bonheur. Cette histoire d'amour ressemble en quelque sorte à celle des Deux pigeons de La Fontaine:
"Deux pigeons s'aimaient d'amour tendre
L'un d'eux s'ennuyant au logis
Fut assez fou pour entreprendre
Un voyage en lointain pays."77

La décision de voyage était là aussi. Les deux gymnospermes de Queneau se séparaient "sans se dire au revoir" (ibid.), mais les deux pigeons de La Fontaine 
"en pleurant ils se dirent adieu." 78

Après que l'un des deux pigeons ait vécu dans la misère et le danger à cause de sa curiosité, la volatile est revenue au logis:

"Demi-morte et demi-boiteuse,

Droit au logis se retourna." 79

L'histoire s'arrête là, la portée nous est claire, le poète nous l'a donnée au début de ses vers:

"L'absence est le plus grand des maux." 80

Le titre un peu changé du poème quenien, Les deux gymnospermes, ne détourne pas le lecteur attentif de découvrir la relation entre ce poème et celui de La Fontaine. La ressemblance existe apparemment, le mot "gymnosperme" n'était utilisé pour aucune fin que pour doter d'humour le message poétique.

Pour le pastiche, il déforme lui aussi, mais en imitant l'hypotexte. C'est dans ce cas que le style est imité sans que le texte ne soit jamais cité. ${ }^{81}$

Autrement dit, le sujet importe peu et c'est le style du texte imité qui est nécessaire pour le pasticheur. C'est donc un exercice de style dont la visée, le plus souvent ludique, peut se révéler sérieuse: quand on imite un auteur, non seulement on apprend à écrire, mais on se libère aussi des influences que cet auteur pourrait avoir sur son propre style.

Nous présentons cet exemple flagrant dans le poème intitulé Si tu t'imagines et dont le recueil porte le même titre: Claude Debon estime que ce poème est "une variation sur la célèbre Ode à Cassandre, "Mignonne, allons voir si la rose...'. Il ne s'agit pas pour Queneau d'un exercice de style gratuit, mais d'un poème inspiré par une déconvenue sentimentale." 82

L'état d'âme dans lequel Queneau a pu écrire ce poème n'est pas tout à fait différent de celui de Ronsard quand il a écrit son Ode à Cassandre en 1553. le poète de l'ode était pris par cette beauté fleurissante de Cassandre, semblable à la rose avec son teint rayonnant. Le temps et la vieillesse, ennemis de toute beauté, pourraient ternir cet épanouissement de la beauté humaine comme c'est le cas de la rose:

"Puisqu'une telle fleur ne dure

Que du matin jusqu'au soir." ${ }^{83}$

Le poète, admirateur de cette beauté humaine, avait fait un appel à Cassandre:

"Cueillez, cueillez votre jeunesse:

Comme à cette fleur, la vieillesse

Fera ternir votre beauté." ${ }^{84}$ 
A cet appel épicurien, nous trouvons un appel pareil chez Queneau dans son poème. "Mignonne" de Ronsard sera remplacé par "fillette" dans le poème quenien. Queneau emploie de même des expressions servant de cliché chez les poètes:

"que ton teint de rose
ta taille de guêpe
tes mignons biceps
tes ongles d'émail
ta cuisse de nymphe..."

(Si tu t'imagines, L'instant fatal, IV, Si tu t'imagines, p. 121).

Le "teint de rose" résonne dans le vers ronsardien: "Et son teint au vôtre pareil". Les aspects de la vie qu'on peut trouver dans une telle "fillette" sont effaçables à cause de la marche irréversible du temps, de l'approche de la mort et du mouvement cyclique des choses:

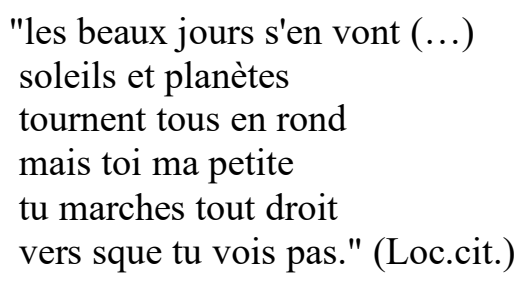

La "marâtre Nature" 85 de Ronsard, cause des maux de la pauvre rose pourrait aussi maltraiter la belle "fillette" de Queneau et la vieillir:

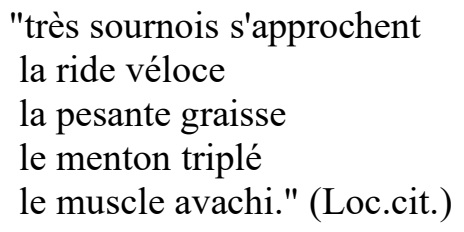

Queneau s'adresse de cet appel à la "fillette" pour qu'elle se hâte de jouir de la vie avant que le temps ne s'en aille:

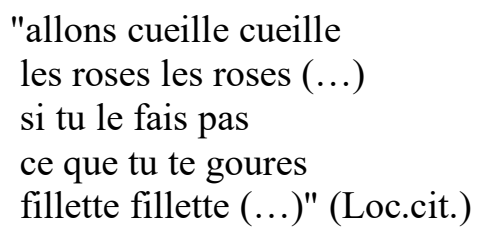

L'appel à Cassandre est semblable à celui de Queneau. Les deux poètes, Ronsard et Queneau, ont presque le même état d'âme troublé et inquiet devant l'approche de la mort, même avec ces élans sensuels passionnés de l'amour de la vie et de la haine de la mort:

"Cueillez, cueillez votre jeunesse: 
Comme à cette fleur la vieillesse

Fera ternir votre beauté." ${ }^{86}$

Donc, Queneau le pasticheur imite ce modèle classique sans vraiment le plagier, il s'en inspire aussi bien le sentiment de peur et d'inquiétude que celui de joie et de plaisir.

Il pleut, c'est le titre d'un autre poème quenien, autre témoin de cette sensibilité poétique géniale. Sous le même titre Apollinaire a créé un poème en calligramme: les mots sont écrits à la japonaise, c'est-à-dire obliquement du haut en bas pour donner la figure de la pluie qui tombe en averse.

Il pleut de Queneau ressemble à celui d'Apollinaire, mais ce premier préfère la forme traditionnelle de l'écriture poétique horizontale. La répétition est le seul moyen du poète par lequel il fait accéder en quelque sorte le poème de l'écrit uniquement au vu, à l'entendu: à la sonorité que produit la reprise des mêmes mots s'ajoute l'image des mots suivis en forme de gouttes d'eau en averse:
"Averse averse averse averse averse averse pluie ô pluie ô pluie! ô pluie ô pluie ô pluie gouttes d'eau gouttes d'eau gouttes d'eau gouttes d'eau parapluie ô parapluie ô paraverse ô! paragouttes d'eau paragouttes d'eau de pluie capuchons pèlerines et imperméables que la pluie est humide et que l'eau mouille et mouille! mouille l'eau mouille l'eau mouille l'eau mouille l'eau et que c'est agréable agréable agréable (...)"

(Si tu t'imagines., Les Ziaux, II, Il pleut, p. 51).

L'effet que produit le poème modèle, celui d'Apollinaire, et que produit le texte d'accueil, celui de Queneau, est le même; les deux donnent la même image de la pluie tombant en abondance, même si les mots se transforment en gouttes d'eau!

La transcription du poème d'Apollinaire, en forme de pluie, est la suivante:

"Il pleut des voix de femmes comme si elles étaient mortes même dans le souvenir c'est vous aussi qu'il pleut merveilleuses rencontres de ma vie ô gouttelettes et ces nuages cabrés se prennent à hennir tout un univers de villes auriculaires écoute s'il pleut tandis que le regret et le dédain pleurent une ancienne musique écoute tomber les liens qui te retiennent en haut et en bas". ${ }^{87}$

La ressemblance entre les deux poèmes, le poème d'Apollinaire et celui de Queneau, est claire: le titre est le même dans les deux; Apollinaire a exploité verticalement la page blanche pour donner de l'image. Pour Queneau, le cas est un peu différent: l'image se crée, chez lui, par la reprise d'un seul mot plusieurs fois. La répétition donne l'influence de la pluie comme c'est le cas pour l'image. Dans le poème d'Apollinaire, l'absence de ponctuation était nécessaire pour la fluidité des 
vers; la possibilité même de l'écriture de l'apostrophe, de la virgule et des points n'était pas tant préconisée dans la poésie d'Apollinaire. Pour lui, la vraie ponctuation $\mathrm{du}$ poème réside dans son rythme, sa pause, ...etc.

Le parodiste, quand il fait un poème selon un modèle quelconque, il ne le fait pas seulement pour le railler, mais il l'admire aussi en s'amusant et en amusant. Pour le pasticheur, de plus que son action est de valeur ludique il l'achève pour une visée cathartique: plus sérieux que le parodiste, le pasticheur cherche en imitant à être conscient du style de l'écrivain qu'il imite, mais d'imposer seulement le sien quand il entreprend l'écriture.

Il se purge de l'idolâtrie et de l'imitation aveugle pour s'inspirer ce qui lui est utile, rejeter le reste et imposer enfin sa propre vision. En cela, la distinction genettienne ${ }^{88}$, entre pastiche et parodie, se montre comme il suit: le pastiche est entendu comme imitation, donc conservation d'un style, et la parodie comme transformation d'un texte.

Voyons dans l'image suivante comment était le poème d'Apollinaire, le modèle qu'a suivi Queneau pour composer son poème: 


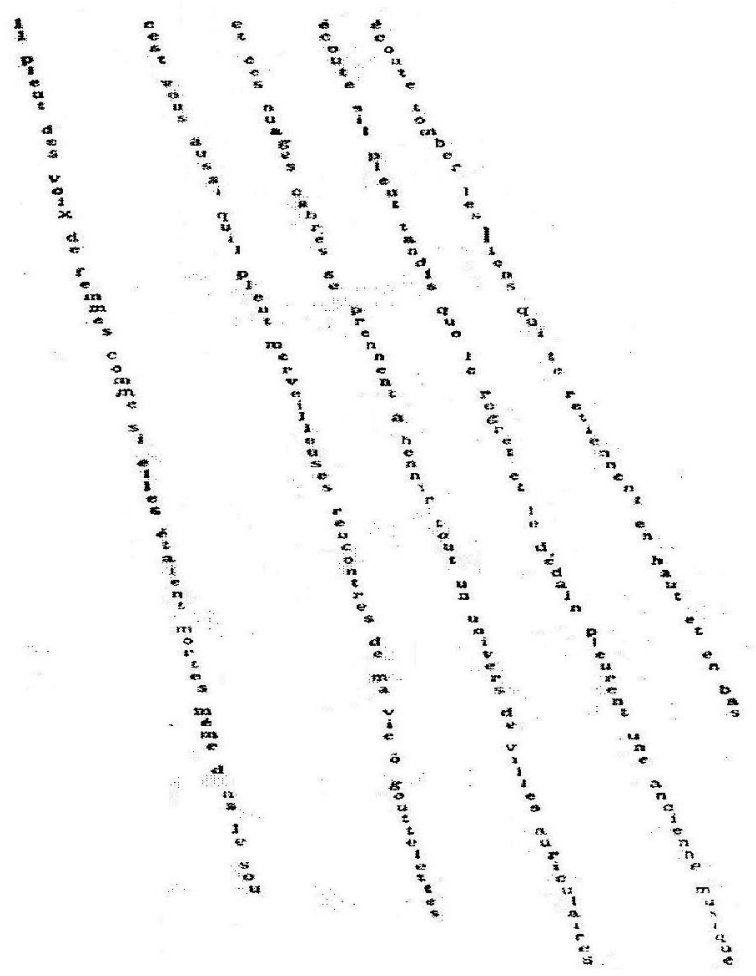

\section{Conclusion}

De ce qui précède, il nous semble que l'œuvre poétique de Queneau n'échappe pas à être un jeu: l'accord implicite entre l'écrivain et son lecteur exige un stock de culture et un effort de mémoire nécessaires à lui fournir une explication logique de cette série d'influences différentes éparpillées ça et là. Ce contrat ludique, pris quand même au sérieux, ajoute au plaisir de la lecture un autre plaisir lié à la contribution du lecteur au mouvement littéraire.

\section{Bibliographie}

\section{I- Corpus}

\begin{tabular}{|l|l|}
\hline QUENEAU Raymond & $\begin{array}{l}\text {-Si tu t'imagines in Euvres complètes, édit. établie par } \\
\text { Claude Debon, Gallimard, nrf, 1989. } \\
\text {-Battre la campagne in Euvres complètes, édit. Etablie } \\
\text { par Claude Debon, Gallimard, Paris 1989. } \\
\text {-"L'Instant fatal" précédé de "Les Ziaux", préface } \\
\text { d'Olivier Magny, postface de Georges Emmanuel } \\
\text { Clancier, édit. Gallimard, coll. Poésie, Paris 1994. } \\
\text {-Chêne et chien, traduit vers l'anglai et commenté par }\end{array}$ \\
\hline
\end{tabular}




\begin{tabular}{|c|c|}
\hline & $\begin{array}{l}\text { Madeleine Velguth, édit. Peter Lang, New York } 1995 \\
\text { (traduit de l'anglais par nous). }\end{array}$ \\
\hline \multicolumn{2}{|l|}{ II- Ouvrages de références } \\
\hline APOLLINAIRE Guillaume & $\begin{array}{l}\text {-Euvres poétiques (préface d'André Billy, édit. Etablie et } \\
\text { annotée par Marcel Adéma et Michel Décaudin), } \\
\text { Gallimard, coll. Bibliothèque de la Pléiade, } 1965 . \\
\text {-Euvres en prose (édition établie par Michel } \\
\text { Décaudin), tome I, édit. La Pléiade, Paris } 1977 .\end{array}$ \\
\hline BAUDELAIRE Charles & $\begin{array}{l}\text {-Les fleurs du mal, Librairie Générale Française, Paris } \\
\text { 1972. }\end{array}$ \\
\hline De VIGNY Alfred & $\begin{array}{l}\text {-Euvres complètes, T. I (poésie et théâtre), Gallimard, } \\
\text { coll. bibliothèque de la Pléiade, } 1986 .\end{array}$ \\
\hline LA FONTAINE Jean de & $\begin{array}{l}\text {-Euvres complètes (édition établie par Jean Marnier, } \\
\text { préface de Pierre Clarac), édit. du Seuil, Paris } 1965 .\end{array}$ \\
\hline \multicolumn{2}{|c|}{ III- Euvres théoriques et de critiques littéraires } \\
\hline BARTHES Roland & -Le Plaisir du texte, édit. du Seuil, 1973. \\
\hline BOUILLAGUET Annick & $\begin{array}{l}\text {-L'Ecriture imitative, Nathan, coll. Littérature, Paris } 1996 . \\
\text {-Seuils, édit. du Seuil, coll. Poétique, Paris, février1987. }\end{array}$ \\
\hline GENETTE Gérard & $\begin{array}{l}\text {-L'esthétique de la réception, Le Conseil Supérieur de la } \\
\text { Culture, le Caire 2002. (traduit par nous de l'arabe). }\end{array}$ \\
\hline ISMAÏL Sami & $\begin{array}{l}\text {-Sémiotikè, Recherches pour une sémanalyse, édit. Seuil, } \\
\text { coll. Points, } 1969 .\end{array}$ \\
\hline KRISTEVA Julia & $\begin{array}{l}\text {-L'Intertextualité "Mémoire de la littérature", } \\
\text { Nathan/HER, Paris } 2001 .\end{array}$ \\
\hline SAMOYAULT Tiphaine & \\
\hline
\end{tabular}

IV-Dictionnaires et encyclopédies

\begin{tabular}{|l|l|}
\hline $\begin{array}{l}\text { AQUIN Michel \& MOLINIÉ } \\
\text { Georges }\end{array}$ & $\begin{array}{l}\text {-Dictionnaire de Rhétorique et de Poétique, édit. Librairie } \\
\text { Générale Française, 1996. } \\
\text {-Larousse élémentaire, Librairie Larousse, Paris } 1956 .\end{array}$ \\
\hline
\end{tabular}

V-Sites internet

http://poesie.webnet.fr/poemes/France/ronsard/105.html; lu le samedi 11 juin 2005.

${ }^{11}$ Julia Kristeva, Sémiotikè, Recherches pour une sémanalyse, Seuil, 1969, p. 85.

2 Ibid., p. 52. 
${ }^{3}$ Loc.cit.

${ }^{4}$ T. Samoyault, L'Intertextualité"Mémoire de la littérature", Nathan/HER, Paris 2001, p. 10.

${ }^{5}$ C.f., ibid., p. 11.

${ }^{6}$ T. Samoyault, L'Intertextualité, op.cit., p. 15.

${ }^{7}$ R. Barthes, Le plaisir du texte, Seuil, 1973, p. 59.

${ }^{8}$ T. Samoyault, op.cit., p. 16.

${ }^{9}$ Ibid., p. 21.

${ }^{10}$ C.f., ibid., p. 24.

${ }^{11} C f$., T. Samoyault, op.cit., p. 28.

${ }^{12} C f$., ibid., pp. 27-28.

${ }^{13} C f$., ibid., p. 29.

${ }^{14} C f$., Sami ISMAÏL, L'esthétique de la réception (traduit par nous de l'arabe), édit. Le

Conseil Supérieur de la Culture, 2002, p. 112.

${ }^{15} \mathrm{Cf}$., loc. cit.

${ }^{16} C f$., loc.cit.

${ }^{17} C f$., ibid., pp. 127-131.

${ }^{18}$ Cité par T. Samoyault, L'intertextualité, op.cit., p. 7.

${ }^{19}$ C.f., Tiphaine Samoyault, op.cit., p. 45.

20 Ibid., pp. 43-45.

${ }^{21}$ Tiphaine Samoyault, op.cit., p. 46.

22 Ibid., p.48.

23 Ibid., p.52.

24 Ibid., p. 24.

${ }^{25}$ loc.cit.

${ }^{26}$ Note citée par T. Samoyault, op.cit., p.24.

27 Ch. Baudelaire, Les fleurs du mal, Mœsta et Errabunda, édit. Librairie Générale Française, 1972, p.85.

28 C.f., C. Debon, Notes et variantes in Euvres complètes, op.cit., p.1134.

29 C. Debon, op.cit., p.1199.

${ }^{30}$ Cité par T. Samoyault, op.cit, p. 53.

31 Ibid., p. 35.

32 C. Debon, Notes et variantes in CEuvres complètes de R. Queneau, op.cit., p. 1135.

33 C.f., C. Debon, Notes et variantes in Euvres completes, op.cit., p. 1387.

34 Ibid., p. 1389.

35 Note citée par C. Debon,, Chronologie in Euvres complètes, op.cit., p. LXXI.

36 T. Samoyault, op.cit., p. 36.

37 Ibid., p. 35.

38 C.f., c. Debon, Notes et variantes in Euvres complètes, op.cit, p. 1153.

39 G.Apollinaire, Alcools in Euvres poétiques, édit. Gallimard, coll. Bibliothèque de la Pléiade, 1965, p. 48.

${ }^{40}$ C. Debon, Notes et variants, op.cit., p. 1153.

${ }^{41}$ Note citée par Larousse Elémentaire, Librairie Larousse, paris 1955.

42 C. Debon, op.cit., p. 1159.

${ }^{43}$ G. Apollinaire, CEuvres en prose, tome I, Gallimard, coll. Bibliothèque de La Pléiade, Paris 1977, p. 527.

${ }^{44}$ C.f., C. Debon, op.cit., p. 1177.

45 . Apollinaire, Alcools in Euvres poétiques, op.cit., p. 64.

46 C. Debon, op.cit., p.1177.

47 c.f., ibid., p. 1390. 
48 Apollinaire, Euvres poétiques, op.cit., p. 70.

49 ibid., p. 71.

${ }^{50}$ Loc.cit.

51 Loc.cit.

52 Ibid., p. 72.

53 Loc.cit.

54 Apollinaire, Euvres poétiques, op.cit., p. 66.

55 C. Debon, op.cit., p.1379.

56 A. de Vigny, Euvres complètes, T. I (poésie et théâtre), Gallimard, coll. bibliothèque de la Pléiade, 1986 , p. 81.

57 C. Debon, op.cit., p. 1395.

58 Baudelaire, Les fleurs du mal, Le voyage VIII, Librairie Générale Française, 1972, p. 177.

59 c.f., T. Samoyault, op.cit., pp. 44-45.

60 Ibid., p. 45.

61 Loc.cit.

62 C. Debon, Notes et variantes, op.cit., p. 1378.

${ }^{63}$ C. Debon, op.cit., p. 1145.

64 T. Samoyault, op.cit., p. 46.

65 c.f., ibid., pp. 46-47.

66 G. Genette, Seuils, édit. du Seuil, coll. Poétique, février 1987, p. 143.

${ }^{67}$ C. Debon, op.cit., p. 1118.

68 Loc.cit.

${ }^{69}$ Cité par T. Samoyault, op.cit., p. 47.

70 Thomas Traherne (1637-1674), était un pasteur anglican.

71 T. Samoyault, L'intertextualité, op.cit., p. 38.

72 C.f., loc.cit.

73 Note citée par le Dictionnaire de Rhétorique et de Poétique, Michèle Aquin et George Molinié, Librairie Générale Française, 1996, p. 68.

74 La Fontaine, Euvres complètes, édit. du Seuil, Paris 1965, p. 78.

75 loc.cit.

76 T. Samoyault, op.cit., p. 38.

77 La Fontaine, Euvres complètes, op.cit., p. 140.

78 Loc.cit.

79 La Fontaine, Euvres complètes, op.cit., p. 140.

80 Loc.cit.

81 c.f., T. Samoyault, op.cit., pp. 38-39.

82 C. Debon, op.cit., p. 1202.

${ }^{83} \mathrm{http} / /$ poesie.webnet.fr/poemes/France/ronsard/105.html ; consulté le samedi, 11 juin 2005.

${ }^{84}$ Loc.cit.

$85 \mathrm{http} / /$ poesie.webnet.fr/poemes/France/ronsard/105.html, op.cit.

${ }^{86} \mathrm{http} / / /$ poesie.webnet.fr/poemes/France/ronsard/105.html, op.cit.

87 G. Apollinaire, CEuvres complètes, édit. Gallimard, Paris 1905, p. 66.

88 C.f., Annick Bouillaguet, L'écriture imitative (pastiche, parodie, collage), Nathan, coll. Littérature, 1996, p. 13. 